\title{
What will help neurobiology take the next step: rejuvennated classics, connectoms, bioinformatics or artificial intelligence?
}

\author{
Igor Bondar \\ IHNA \& NPh RAS, Moscow, Russia \\ bondar@ihna.ru
}

\begin{abstract}
Neurobiology is a rapidly developing field of modern science. New research technologies allow researchers to actively intervene in the work of the whole brain and local neural networks. A true understanding of the fundamental principles of the brain functioning is possible only by combination of systematic approach in the analysis of complex behavior and detailed information on neuronal functions.
\end{abstract}

Keywords - extracellular recordings, connectomics, optogenetic approach, artificial intelligence

The central issue in neurobiology remains the understanding of how the activity of single neurons, combined into distributed neural networks, provides animals with the ability to adapt to rapidly changing environmental conditions using a specific behavioral pattern. Adaptive behavior can be observed in simply organized living beings who lack a highly developed nervous system, but have the opportunity to feel changes in the physical or chemical properties of the environment, as well as the ability to actively change position in space.

Considering the case of the evolution of the visual system, it was shown that the principle of "division of labor" underlie evolutionary changes [1]. As a result specialized cells emerging in the functional space between the sensitive part of the nervous system and motoneurons that provide response to the stimulus. An "interneuronal explosion" gave the nervous system the capabilities of a finer analysis of changes in environment, but at the same time it complicated the life for modern neurobiologists who risk being buried under the rubble of experimental data.

Currently, neurobiology dominated by an interventionist approach, which involves active intervention in the brain function. It opposed by a systematic approach, whose defenders propose a deeper analysis of animal behavior [2]. This polarization in modern brain research is associated with the progress of sophisticated scientific technologies that provide modern neuroscientists with a wealth of data.

The development of computer technology has given new life to classical brain research methods. The technique for recording extracellular potentials, which was invented about 100 years ago, is used today to record the activity of a large number of single neurons simultaneously. Over the years, the study of the properties of single neurons has been a key research technology in the works that were awarded two times by Nobel Prize. A study of the properties of neuron detectors in the primary visual cortex of a cat's brain and the place cells in a rat's hippocampus has enriched an understanding of fundamental principles organizing the work of the whole brain.
Currently, chronically implanted multiple microelectrodes or special matrices can be used in conjunction with brain activity imaging methods, which makes it possible to determine the degree of involvement of the activity of individual cells in the functioning of distributed neural ensembles. Long-term registrations provide an opportunity to observe plastic changes in the activity of nerve cells associated with the acquisition of experience and learning. Special value this method has for the long-term registration of neurons in experiments connected with the studies of the relationship between neuronal activity and the analysis of sensory information. In this case formation of the internal representation of environmental objects can be investigated [3]. In our experiments we have shown that the prototype, the "averaged image" that forms in specialized parts of the brain, can play a central role in recognition of faces. Thus, updated classical methods can still provide researchers with valuable experimental data. Moreover, methodological approaches tested in animal experiments can become the basis for creating safe and reliable invasive brain-machine interfaces that will provide the ability to restore impaired brain functions of patients [4].

Along with classical methods, new approaches also appear. Some time ago, the idea began to circulate in the research environment that a true understanding of brain functions is possible by detailed description of all its connections. Currently, active work is underway to create a digitalized description of neural networks with a resolution level of a single synapse [5]. Connectoms allow to accurately describe the ultrastructure of the brain, but they are extremely time-consuming and requires large amounts of computing power. The main problem is that at the conceptual level we still do not have an understanding for algorithms that will allow us to move from structure to function. Given that at all levels of the organization, the nervous system demonstrates an amazing ability for constant changes, a detailed "snapshot" of the structure can have only limited value for understanding of fundamental principles of the brain function.

Strengthening the position of the interventionist approach in neurobiology has become possible in connection with the development of genetic and molecular methods. Genome sequencing becomes available for different model organisms, which allows to purposefully introduce new information into them, affecting the functional properties of neurons. The optogenetic approach uses the full power of bioinformatics for targeted transfection of subpopulations of neurons [6]. Subsequent local light exposure of nerve tissue gaves researchers the possibility to change the functionality of neural networks. Optogenetic experiments not only shed light on the fundamental aspects of the brain function, but also allow to restore its lost performance [7]. 
Artificial intelligence has two possible points of application in modern neurobiology. In some labor-intensive research technologies, it can be a working tool for solving specific problems. Automated analysis of the results of structural and functional brain imaging becomes more efficient when using artificial intelligence. The combination of artificial intelligence with the collective work of a large number of proofreaders forms a unique symbiosis of a person and a computer program in which both sides get advantages [5]. On the other hand, understanding the basics of natural neural ensembles will allow us to implement this knowledge into the functional architecture of artificial neural networks, which will become the basis for creating new assistive technologies that increase the efficiency of human activities. While research in the field of artificial intelligence is based more on a mathematical apparatus, an important task for neurobiology could be to provide this direction of science with content [8].

Successes in various fields of neurobiology in the context of the general development of knowledge about living things allow us to look to the future with optimism. However, it is extremely important to remember that only a systematic approach to the analysis of the entire array of experimental data will allow us to achieve such an understanding of the brain, which in the future will allow us to "repair" it.

\section{ACKNOWLEDGMENT}

Supported by the RFBR (19-015-00349) und funds within the state assignment of Ministry of Education and Science of the Russian Federation for 2019-2021 (AAAA-A17117092040002-6)

\section{REFERENCES}

[1] Arendt D. et al. (2009) The 'division of labour' model of eye evolution.

[2] Philos Trans R Soc Lond B Biol Sci. 364(1531):2809-17. doi: 10.1098/rstb.2009.0104.

[3] Krakauer J.W. et al. (2017) Neuroscience Needs Behavior: Correcting a Reductionist Bias. Neuron. 93(3):480-490. doi: 10.1016/j.neuron.2016.12.041

[4] Leopold D.A. et al. (2006) Norm-based face encoding by single neurons in the monkey inferotemporal cortex. Nature. 442(7102):5725 .

[5] Lebedev M.A., Nicolelis M.A. (2017) Brain-Machine Interfaces: From Basic Science to Neuroprostheses and Neurorehabilitation. Physiol Rev. 97(2):767-837

[6] Lee K. et al. (2019) Convolutional nets for reconstructing neural circuits from brain images acquired by serial section electron microscopy. Curr. Opin. Neurobiol. 55:188-198. doi: 10.1016/j.conb.2019.04.001.

[7] Lamprecht R (2019). Regulation of signaling proteins in the brain by light. Prog Neurobiol. 180:101638. doi: 10.1016/j.pneurobio.2019.101638

[8] Simon C.J. et al. (2020) Opsins for vision restoration. Biochem. Biophys. Res. Commun. pii: S0006-291X(20)30074-7. doi: 10.1016/j.bbrc.2019.12.117

[9] Savage N. (2019) How AI and neuroscience drive each other forwards. Nature. 571(7766):S15-S17. doi: 10.1038/d41586-019-02212-4. 